\title{
An Approach for Combining Content-based and Collaborative Filters
}

\author{
Qing Li \\ Dept. of Computer Sciences \\ Kumoh National Institute of Technology \\ Kumi, kyungpook, 730-701,South Korea \\ liqingese.Kumoh.ac.kr
}

\author{
Byeong Man Kim \\ Dept. of Computer Sciences \\ Kumoh National Institute of Technology \\ Kumi, kyungpook, 730-701,South Korea \\ bmkimese.Kumoh.ac.kr
}

\begin{abstract}
In this work, we apply a clustering technique to integrate the contents of items into the item-based collaborative filtering framework. The group rating information that is obtained from the clustering result provides a way to introduce content information into collaborative recommendation and solves the cold start problem. Extensive experiments have been conducted on MovieLens data to analyze the characteristics of our technique. The results show that our approach contributes to the improvement of prediction quality of the item-based collaborative filtering, especially for the cold start problem.
\end{abstract}

\section{Introduction}

There are two dominant research paradigms of information filtering: content-based and collaborative filtering. Content-based filtering selects the right information for users by comparing representations of searching information to representations of contents of user profiles which express interests of users. Content-based information filtering has proven to be effective in locating textual items relevant to a topic using techniques, such as Boolean queries (Anick et al., 1990; Lee et al., 1993; Verhoeff et al., 1961), vector-space queries (Salton and Buckley, 1998), probabilistic model (Robertson and Sparck, 1976), neural network (Kim and Raghavan, 2000) and fuzzy set model (Ogawa et

This work was supported by Korea Research Foundation Grant (KRF-2002-041-D00459). al., 1991). However, content-based filtering has some limitations:

- It is hard for content-based filtering to provide serendipitous recommendations, because all the information is selected and recommended based on the content.

- It is hard for novices to use content-based systems effectively.

Collaborative filtering is the technique of using peer opinions to predict the interests of others. A target user is matched against the database to discover neighbors, who have historically had similar interests to target user. Items that neighbors like are then recommended to the target user. The Tapestry text filtering system, developed by Nichols and others at the Xerox Palo Alto Research Center (PARC), applied collaborative filtering (Douglas, 1993; Harman, 1994). The GroupLens project at the University of Minnesota is a popular collaborative system. Collaborative systems have been widely used in so many areas, such as Ringo system recommends music albums (Upendar and Patti, 1995), MovieLens system recommends movies, Jeter system recommends jokes (Gupta et al., 1999) and Flycasting recommends online radio (Hauver, 2001).

Collaborative filtering system overcomes some limitations of content-based filtering. The system can suggest items (the things to be recommended, such as books, music etc.) to users and recommendations are based on the ratings of items, instead of the contents of the items, which can improve the quality of recommendations. Although collaborative filtering has been successfully used in both research and practice, there still remain some challenges for it as an efficient information filtering. 
- Cold start problem, where recommendations are required for items that no user has yet rated.

- Although collaborative filtering can improve the quality of recommendations based on the user ratings, it completely denies any information that can be extracted from contents.

It is obvious that the content-based filtering does not suffer the above problems. So it is a natural way to combine them in order to achieve a better performance of filtering, and take the advantages of each.

The rest of the paper is organized as follows. The next section provides a brief describing of related work. In section 3, we present the detail algorithmic components of our approach, and look into the methods of grouping items, calculating the similarities between items and solving the cold start problem. Section 4 describes our experimental work. It provides details of our data sets, evaluation metrics, results of our experiment and discussion of the results. The final section provides some concluding remarks.

\section{Related work}

Proposed approaches to hybrid system, which combines content-based and collaborative filters together, can be categorized into two groups.

One group is the linear combination of results of collaborative and content-based filtering, such as systems that are described by Claypool (1999) and Wasfi (1999). ProfBuilder (Wasfi, 1999) recommends web pages using both content-based and collaborative filters, and each creates a recommendation list without combining them to make a combined prediction. Claypool (1999) describes a hybrid approach for an online newspaper domain, combining the two predictions using an adaptive weighted average: as the number of users accessing an item increases, the weight of the collaborative component tends to increase. But how to decide the weights of collaborative and contentbased components is unclearly given by the author.

The other group is the sequential combination of content-based filtering and collaborative filtering. In this system, firstly, content-based filtering algorithm is applied to find users, who share similar interests. Secondly, collaborative algorithm is applied to make predictions, such as RAAP (Delgado et al., 1998) and Fab filtering systems
(Balabanovic and Shoham, 1990). RAAP is a content-based collaborative information filtering for helping the user to classify domain specific information found in the WWW, and also recommends these URLs to other users with similar interests. To decide the similar interests of users is using scalable Pearson correlation algorithm based on web page category. Fab system, which uses contentbased techniques instead of user ratings to create profiles of users. So the quality of predictions is fully depended on the content-based techniques, inaccurate profiles result in inaccurate correlations with other users and thus make poor predictions.

As for collaborative recommendation, there are two ways to calculate the similarity for clique recommendation - item-based and user-based. Sarwar (Sarwar et al, 2001) has proved that item-based collaborative filtering is better than user-based collaborative filtering at precision and computation complexity.

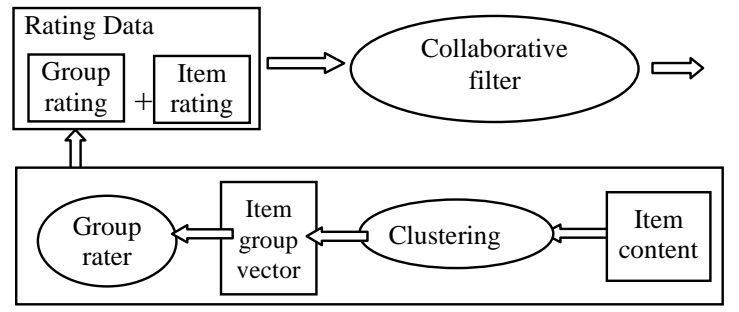

Figure1. Overview of the our approach

\section{Overview of our approach}

In this paper, we suggest a technique that introduces the contents of items into the item-based collaborative filtering to improve its prediction quality and solve the cold start problem. Shortly, we call the technique ICHM (Item-based Clustering Hybrid Method).

In $I C H M$, we integrate the item information and user ratings to calculate the item-item similarity. Figure 1 shows this procedure. The detail procedure of our approach is described as follows:

- Apply clustering algorithm to group the items, then use the result, which is represented by the fuzzy set, to create a grouprating matrix.

- Compute the similarity: firstly, calculate the similarity of group-rating matrix using adjusted-cosine algorithm, then calculate the similarity of item-rating matrix using Pearson correlation-based algorithm. At last, the 
total similarity is the linear combination of the above two.

- Make a prediction for an item by performing a weighted average of deviations from the neighbour's mean.

\subsection{Group rating}

The goal of grouping ratings is to group the items into several cliques and provides content-based information for collaborative similarity calculation.

Each item has it's own attribute features, such as movie item, which may have actor, actress, director, genre, and synopsis as its attribute features. Thus, we can group the items based on them.

The algorithm that is applied for grouping ratings is derived from K-means Clustering Algorithm (Han and Kamber, 2000). The difference is that we apply the fuzzy set theory to represent the affiliation between object and cluster. As shown in Figure 2, firstly, items are grouped into a given number of clusters. After completion of grouping, the probability of one object $j$ (here one object means one item) to be assigned to a certain cluster is calculated as follows.

$$
\operatorname{Pr} o(j, k)=1-\frac{C S(j, k)}{\operatorname{Max} C S(i, k)}
$$

where $\operatorname{Pr} o(j, k)$ means the probability of object $j$ to be assigned to cluster $k$; The $C S(j, k)$ means the function to calculate the counter-similarity between object $j$ and cluster $k ; \operatorname{Max} C S(i, k)$ means the maximum counter-similarity between an object and cluster $k$.

Input : the number of clusters $k$ and item attributes Output: a set of $k$ clusters that minimizes the squarederror criterion, and the probability of each item to be assigned to each cluster center, which are represented as a fuzzy set.

(1) Arbitrarily choose $k$ objects as the initial cluster centers

(2) Repeat (a) and (b) until no change

(a) $(\mathrm{Re})$ assign each object to the cluster to which the object is the most similar, based on the mean value of the objects in the cluster

(b) Update the cluster means, i.e., calculate the mean value of the objects of each cluster;

(3) Compute the probability between objects and each cluster center.

Figure 2. Algorithm for grouping ratings
The counter-similarity $C S(j, k)$ can be calculated by Euclidean distance or Cosine method.

\subsection{Similarity computation}

As we can see, after grouping the items, we get a new rating matrix. We can use the item-based collaborative algorithm to calculate the similarity and make the predictions for users.

There are many ways to compute the similarity. In our approach, we use two of them, and make a linear combination of their results.

\subsubsection{Pearson correlation-based similarity}

The most common measure for calculating the similarity is the Pearson correlation algorithm. Pearson correlation measures the degree to which a linear relationship exists between two variables. The Pearson correlation coefficient is derived from a linear regression model, which relies on a set of assumptions regarding the data, namely that the relationship must be linear, and the errors must be independent and have a probability distribution with mean 0 and constant variance for every setting of the independent variable (McClave and Dietrich, 1998).

$$
\operatorname{sim}(k, l)=\frac{\operatorname{cov}(k, l)}{\sigma_{k} \sigma_{l}}=\frac{\sum_{u=1}^{m}\left(R_{u, k}-\bar{R}_{k}\right)\left(R_{u, l}-\bar{R}_{l}\right)}{\sqrt{\sum_{u=1}^{m}\left(R_{u, k}-\bar{R}_{k}\right)^{2}} \sqrt{\sum_{i=1}^{m}\left(R_{u, l}-\bar{R}_{l}\right)^{2}}}
$$

where $\operatorname{sim}(k, l)$ means the similarity between item $k$ and $l ; m$ means the total number of users, who rated on both item $k$ and $l ; \bar{R}_{k}, \bar{R}_{l}$ are the average ratings of item $k$ and $l$, respectively; $R_{u, k}, R_{u, l}$ mean the rating of user $u$ on item $k$ and $l$ respectively.

\subsubsection{Adjust cosine similarity}

Cosine similarity once has been used to calculate the similarity of users but it has one shortcoming. The difference in rating scale between different users will result in a quite different similarity. For instance, if Bob only rates score 4 on the best movie, he never rates 5 on any movie; and he rates 1 on the bad movie, instead of the standard level score 2. But Oliver always rates according to the standard level. He rates score 5 on the best movie, and 2 on the bad movie. If we use traditional cosine similarity, both of them are quite different. The adjusted cosine similarity (Sarwar et al., 2001) was provided to offset this drawback. 


$$
\operatorname{sim}(k, l)=\frac{\sum_{u=1}^{m}\left(R_{u, k}-\bar{R}_{u}\right)\left(R_{u, l}-\bar{R}_{u}\right)}{\sqrt{\sum_{u=1}^{m}\left(R_{u, k}-\bar{R}_{u}\right)^{2}} \sqrt{\sum_{u=1}^{m}\left(R_{u, l}-\bar{R}_{u}\right)^{2}}}
$$

where $\operatorname{sim}(k, l)$ means the similarity between item $k$ and $l ; m$ means the total number of users, who rates on both item $k$ and $l ; \bar{R}_{u}$ are the average ratings of user $u ; R_{u, k}, R_{u, l}$ mean the rating of user $u$ on item $k$ and $l$ respectively.

\subsubsection{Linear combination of similarity}

Due to difference in value range between itemrating matrix and group-rating matrix, we use different methods to calculate the similarity. As for item-ratings matrix, the rating value is integer; As for group-rating matrix, it is the real value ranging from 0 to 1 . The natural way is to enlarge the continuous data range from [0 1] to [1 5] or reduce the discrete data range from [1 5] to [0 1] and then apply Pearson correlation-based algorithm or adjusted cosine algorithm to calculate similarity. We call this enlarged ICHM. We also propose another method: firstly, use Pearson correlation-based algorithm to calculate the similarity from item-rating matrix, and then calculate the similarity from group-rating matrix by adjusted cosine algorithm, at last, the total user similarity is linear combination of the above two, we call this combination ICHM.

$$
\operatorname{sim}(k, l)=\operatorname{sim}(k, l)_{\text {item }} \times(1-c)+\operatorname{sim}(k, l)_{\text {group }} \times c
$$

where $\operatorname{sim}(k, l)$ means the similarity between item $k$ and $l ; c$ means the combination coefficient; $\operatorname{sim}(k, l)_{\text {item }}$ means that the similarity between item $k$ and $l$, which is calculated from item-rating matrix; $\operatorname{sim}(k, l)_{\text {group }}$ means that the similarity between item $k$ and $l$, which is calculated from grouprating matrix.

\subsection{Collaborative prediction}

Prediction for an item is then computed by performing a weighted average of deviations from the neighbour's mean. Here we use top $N$ rule to select the nearest $N$ neighbours based on the similarities of items. The general formula for a prediction on item $k$ of user $u$ (Resnick et al., 1994) is:

$$
P_{u, k}=\bar{R}_{k}+\frac{\sum_{i=1}^{n}\left(R_{u, i}-\bar{R}_{i}\right) \times \operatorname{sim}(k, i)}{\sum_{i=1}^{n}|\operatorname{sim}(k, i)|}
$$

where $P_{u, k}$ represents the predication for the user $u$ on item $k ; n$ means the total neighbours of item $k ; R_{u, i}$ means the user $u$ rating on item $i ; \bar{R}_{k}$ is the average ratings on item $k ; \operatorname{sim}(k, i)$ means the similarity between item $k$ and its' neighbour $i ; \bar{R}_{i}$ means the average ratings on item $i$.

\subsection{Cold start problem}

In traditional collaborative filtering approach, it is hard for pure collaborative filtering to recommend a new item to user since no user made any rating on this new item. However, in our approach, based on the information from group-rating matrix, we can make predictions for the new item. In our experiment, it shows a good recommendation performance for the new items. In Equation $5, \bar{R}_{k}$ is the average rating of all ratings on item $k$. As for the new item, no user makes any rating on it, $\bar{R}_{k}$ should be the zero. Since $\bar{R}_{k}$ is the standard baseline of user ratings and it is zero, it is unreasonable for us to apply Equation 5 to new item. Therefore, for a new item, we use the $\bar{R}_{\text {neighbors }}$, the average rating of all ratings on the new item's nearest neighbour instead of $\bar{R}_{k}$, which is inferred by the group-rating matrix.

\subsection{A scenario of our approach}

- Users:

Number of users: three

User name: Tom, Jack, and Oliver

- Items:

Item category: movie

Number of items: five

Title of items: Gone with the Wind, Pearl Harbour, Swordfish, Hero, The Sound of Music

- Ratings: 1 5 integer score

Too bad:1 Bad:2 Common:3 Good:4 too good:5

Table 1: Item-rating

\begin{tabular}{|c|c|c|c|}
\hline & Tom & Jack & Olive \\
\hline Gone with the Wind & 5 & 3 & \\
\hline Swordfish & 5 & 2 & 4 \\
\hline Pearl Harbour & 2 & 5 & \\
\hline Hero & 4 & 2 & \\
\hline The Sound of Music & & & \\
\hline
\end{tabular}

Table 2. Group-rating

\begin{tabular}{|c|c|c|}
\hline & Cluster1 & Cluster2 \\
\hline Gone with the Wind & $98 \%$ & $0.13 \%$ \\
\hline Swordfish & $100 \%$ & $0.02 \%$ \\
\hline Pearl Harbour & $1.0 \%$ & $95 \%$ \\
\hline
\end{tabular}




\begin{tabular}{|c|c|c|}
\hline Hero & $95 \%$ & $1.2 \%$ \\
\hline The Sound of Music & $0.12 \%$ & $98 \%$ \\
\hline
\end{tabular}

The following is a procedure of our approach.

- Based on the item contents, such as movie genre, director, actor, actress, even synopsis, we apply clustering algorithm to group the items. Here, we use fuzzy set to represent the clustering result. Assume the result is as follows: Cluster 1: \{Gone with the Wind (98\%), Swordfish (100\%), Pearl Harbour (1.0\%), Hero (95\%), The Sound of Music $(0.12 \%)\}$, Cluster 2: \{Gone with the Wind (0.13\%), Swordfish (0.02\%), Pearl Harbour (95\%), Hero (1.2\%), The Sound of Music $(98 \%)\}$, the number in the parenthesis following the movie name means the probability of the movie belonging to the cluster.

- We use group-rating engine to make a group-rating matrix. As Table 2 shows. Then combine the group-rating matrix and item-rating matrix to form a new rating matrix.

- Now, we can calculate the similarity between items based on this new unified rating data matrix. The similarity between items consists of two parts. The first part calculates the similarity based on user ratings, using the Pearson correlation-based algorithm. The second part calculates the similarity based on the clustering result by using adjusted cosine algorithm. The total similarity between items is the linear combination of them. For example, when we calculate the similarity between Gone with the Wind and Swordfish, firstly, $\operatorname{sim}(\mathrm{G}, \mathrm{S})_{\text {item }}$ and $\operatorname{sim}(\mathrm{G}, \mathrm{S})_{\text {group }}$ are calculated based on Equation 2 and 3 separately.

$$
\begin{aligned}
& \operatorname{sim}(\mathrm{G}, \mathrm{S})_{\mathrm{item}}=\frac{(5-4) \times(5-3.5)+(3-4) \times(2-3.5)}{\sqrt{(5-4)^{2}+(3-4)^{2}} \times \sqrt{(5-3.5)^{2}+(3.5-2)^{2}}}=1 \\
& \operatorname{sim}(\mathrm{G}, \mathrm{S})_{\text {group }}= \\
& \frac{(0.98-0.59) \times(1-0.59)+(0.013-0.39) \times(0.002-0.39)}{\sqrt{(0.98-0.59)^{2}+(0.013-0.39)^{2}} \sqrt{(1-0.59)^{2}+(0.002-0.39)^{2}}} \\
& =0.9999
\end{aligned}
$$

Secondly, $\operatorname{sim}(\mathrm{G}, \mathrm{S})$ is calculated based on Formula 4, here the combination coefficient is 0.4 .

$\operatorname{sim}(\mathrm{G}, \mathrm{S})=1 \times(1-0.4)+0.9999 \times 0.4=0.9999$
- Then, predictions for items are calculated by performing a weighted average of deviations from the neighbour's mean.

In the example, we can observe, the item - The Sound of Music, which no one make any rating on, can be treated as a new item. In traditional itembased collaborative method, which makes prediction only based on item-based matrix (Table 1), it is impossible to make predictions on this item. However, in our approach, we can make prediction for users, based on group rating (Table 2).

From the description of our approach, we can observe that this approach can fully realize the strengths of content-based filtering, mitigating the effects of the new user problem. In addition, when calculating the similarity, our approach considers the information not only from personal tastes but

\begin{tabular}{|c|c|c|c|c|c|c|c|c|}
\hline \multicolumn{9}{|c|}{ UCHM } \\
\hline & \multicolumn{2}{|c|}{ Movie 1} & \multicolumn{2}{|c|}{ Movie 2} & \multicolumn{2}{|c|}{ Movie 3} & Cluster 1 & Cluster 2 \\
\hline User 1 & \multicolumn{2}{|c|}{$\ldots$} & \multicolumn{2}{|c|}{$\ldots$} & \multicolumn{2}{|c|}{$\ldots$} & $\ldots$ & $\ldots$ \\
\hline User 2 & \multicolumn{2}{|c|}{$\ldots$} & \multicolumn{2}{|c|}{$\ldots$} & \multicolumn{2}{|c|}{$\ldots$} & $\ldots$ & $\ldots$ \\
\hline \multirow[t]{3}{*}{ User 3} & \multicolumn{2}{|c|}{$\ldots$} & \multicolumn{2}{|c|}{$\ldots$} & \multicolumn{2}{|c|}{$\ldots$} & $\cdots$ & $\ldots$ \\
\hline & \multicolumn{6}{|c|}{$\begin{array}{c}\text { Item-rating Matrix } \\
\end{array}$} & \multicolumn{2}{|c|}{\begin{tabular}{c|c} 
& \\
Group-rating Matrix
\end{tabular}} \\
\hline & \multicolumn{7}{|c|}{ ICHM } & \\
\hline \multicolumn{2}{|c|}{ ICHM } & \multicolumn{2}{|c|}{ User 1} & \multicolumn{2}{|c|}{ User 2} & User 3 & 3 Cluster 1 & 1 Cluster 2 \\
\hline \multicolumn{2}{|c|}{ Movie 1} & & $\ldots$ & \multicolumn{2}{|c|}{$\cdots$} & $\ldots$ & $\ldots$ & $\ldots$ \\
\hline \multicolumn{2}{|c|}{ Movie 2} & & $\ldots$ & \multicolumn{2}{|c|}{$\ldots$} & $\ldots$ & $\ldots$ & $\ldots$ \\
\hline \multicolumn{2}{|c|}{ Movie 3} & & $\ldots$ & \multicolumn{2}{|c|}{$\ldots$} & $\cdots$ & $\cdots$ & $\cdots$ \\
\hline
\end{tabular}
also from the contents, which provides a latent ability for better prediction and makes serendipitous recommendation.

\section{$3.6 \quad \mathrm{UCHM}$}

Figure 3. UCHM \& ICHM

Clustering technique not only can be applied to item-based collaborative recommenders but also can be applied to user-based collaborative recommenders. Shortly we call the late one UCHM (User-based Clustering Hybrid Method)

In $U C H M$, clustering is based on the attributes of user profiles and clustering result is treated as items. However, in ICHM, clustering is based on the attributes of items and clustering result is treated as users, as Figure 3 shows.

In Combination UCHM, we apply Equation 2 to calculate the similarity in user-rating matrix, and 
Equation 3 to calculate the similarity in grouprating matrix. Then make a linear combination of them. When we apply Equation 2 and 3 to $U C H M$, $k$ and $l$ mean the user and $u$ means the item, instead the original meaning.

As for $U C H M$, clustering is based on the user profiles. User profiles indicate the information needs or preferences on items that users are interested in. A user profile can consist of several profile vectors and each profile vector represents an aspect of his preferences, such as movie genre, director, actor, actress and synopsis. The profile vectors are automatically constructed from rating data by the following simple equation.

$$
A=m / n
$$

where, $n$ is the number of items whose ranking value is lager than a given threshold, $m$ is the number of items containing attribute $A$ among $n$ items and its ranking is larger than threshold. In our experiment, we set the value of the threshold as 3 . For example, in Section 3.5, Tom makes ratings on four movies, and three of them lager than the threshold 3. From the genre information, we know Gone with the Wind belongs to love genre, swordfish and Hero belong to action genre. So Tom's profile is as follows. Tom $\{$ love $(1 / 3)$, action $(2 / 3)\}$.

\section{Experimental evaluations}

\subsection{Data set}

Currently, we perform experiment on a subset of movie rating data collected from the MovieLens web-based recommender. The data set contained 100,000 ratings from 943 users and 1,682 movies, with each user rating at least 20 items. We divide data set into a training set and a test data set.

\subsection{Evaluation metrics}

MAE (Mean Absolute Error) has widely been used in evaluating the accuracy of a recommender system by comparing the numerical recommendation scores against the actual user ratings in the test data. The MAE is calculated by summing these absolute errors of the corresponding ratingprediction pairs and then computing the average.

$$
M A E=\frac{\sum_{u=1}^{n}\left|P_{u, i}-R_{u, i}\right|}{n}
$$

where $P_{u, i}$ means the user $u$ prediction on item $i$; $R_{u, i}$ means the user $u$ rating on item $i$ in the test data; $n$ is the number of rating-prediction pairs between the test data and the prediction result. The lower the MAE, the more accurate.

\subsection{Behaviours of our method}

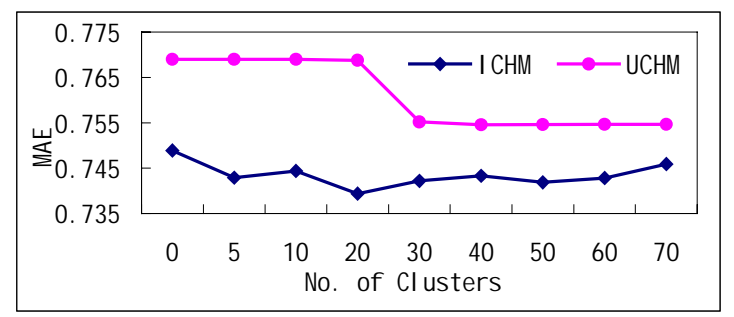

Figure 4. Sensitivity of the cluster size

We implement group-rating method described in section 3.1 and test them on MovieLens data with the different number of clusters. Figure 4 shows the experimental results. It can be observed that the number of clusters does affect the quality of prediction, no matter in UCHM or ICHM.

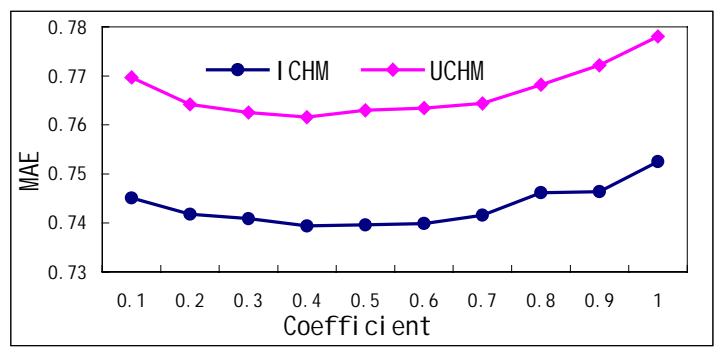

Figure 5. Coefficient

In order to find the optimal combination coefficient $\mathrm{c}$ in the Equation 4, we conducted a series of experiments by changing combination coefficient from 0 to 1 with a constant step 0.1 . Figure 5 shows that when the coefficient arrives at 0.4 , an optimal recommendation performance is achieved.

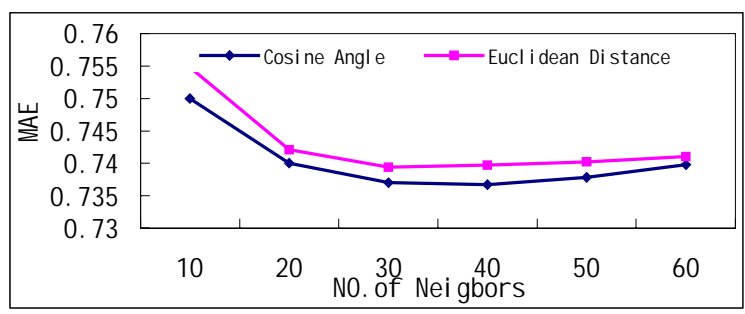

Figure 6. Grouping items

As described in Section 3.2, our grouping ratings method needs to calculate similarity between 
objects and clusters. So, we try two methods - one is Euclidean distance and the other cosine angle. It can be observed in Figure 6 that the approach of cosine angle method has a trend to show better performance than the Euclidean Distance method, but the difference is negligible.

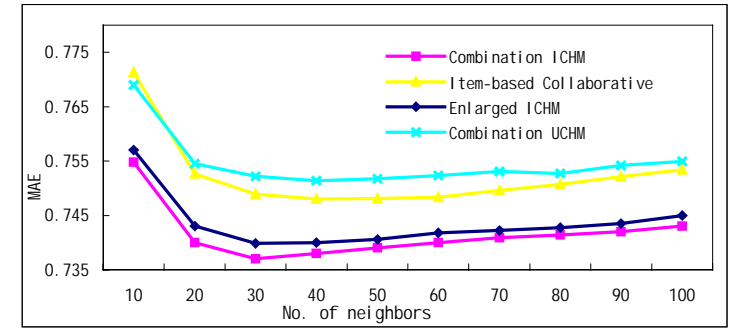

Figure 7. Comparison

From the Figure 7, it can be observed that the performance of combination ICHM is the best, and the second is the enlarged ICHM, which is followed by the item-based collaborative method, the last is UCHM (User-based Clustering Hybrid Method) which applies the clustering technique described in Section 3 to user-based collaborative filtering, where user profiles are clustered instead of item contents.

We also can observe that the size of neighbourhood does affect the quality of prediction (Herlocker et al., 1999). The performance improves as we increase the neighbourhood size from 10 to 30 , then tends to be flat.

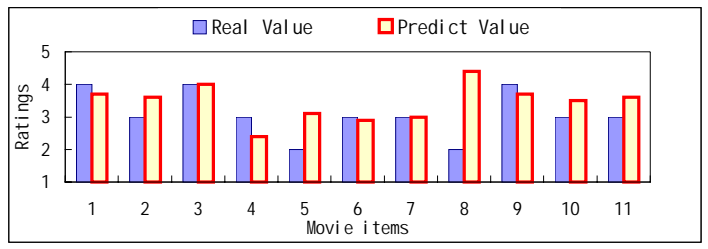

Figure 8. Cold start problem

Table 3. MAE of new item

\begin{tabular}{|c|c|c|c|c|c|c|}
\hline & 10 & 20 & 30 & 40 & 50 & 100 \\
\hline MAE & 0.743 & 0.755 & 0.812 & 0.732 & 0.762 & 0.757 \\
\hline
\end{tabular}

As for cold start problem, we choose the items from the training data set and delete all the ratings of those items, thus we can treat them as new items. First, we randomly selected item No.946. In the test data, user No.946 has 11 ratings, which is described by bar real value in Figure 8. We can observe that the prediction for a new item can partially reflect the user preference. To generalize the observation, we randomly select the number of items from 10 to 50 with the step of 10 and 100 from the test data, and delete all the ratings of those items and treat them as new items. Table 3 shows that ICHM can solve the cold start problem.

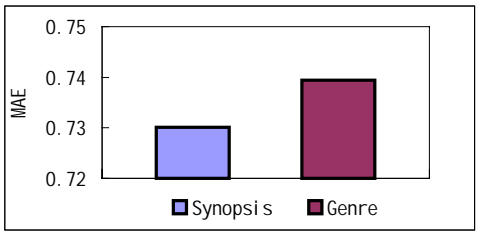

Figure 9. Item attribute

When we apply clustering method to movie items, we use the item attribute - movie genre. However, our approach can consider more dimension of item attribute, such as actor, actress, and director, even the synopsis. In order to observe the effect of the high dimension item attributes, we collect the 100 movie synopsis from Internet Movie Database (http://www.imdb.com) to provide attribute information for clustering movies. In our experiment, it shows that the correct attributes of movies can further improve the performance of recommender system, as Figure 9 shows.

\subsection{Our method versus the classic one}

Although some hybrid recommender systems have already exited, it is hard to make an evaluation among them. Some systems (Delgado et al., 1998) use Boolean value (relevant or irrelevant) to represent user preferences, while others use numeric value. The same evaluation metrics cannot make a fair comparison. Further more, the quality of some systems depends on the time, in which system parameters are changed with user feedback (Claypool et al., 1999), and Claypool does not clearly describe how to change the weight with time passed. However, we can make a simple concept comparison. In Fab system, the similarity for prediction is only based on the user profiles. As for UCHM, which groups the content information of user profiles and uses user-based collaborative algorithm instead of ICHM, the impact of combination coefficient can be observed in Figure 5. In UCHM, when the value of coefficient equals to 1 , it describes condition that Fab applied, which means the similarity between users is only calculated from the group-rating matrix. In that condition, the MAE shows the worst quality of recommendation. 


\section{Conclusions}

We apply clustering technique to the item content information to complement the user rating information, which improves the correctness of collaborative similarity, and solves the cold start problem. Our work indicates that the correct application of the item information can improve the recommendation performance.

\section{References}

Anick, P. G., Brennan, J. D., Flynn, R. A., Hanssen, D. R., Alvey, B. and Robbins, J.M.. 1990. A Direct Manipulation Interface for Boolean Information Retrieval via Natural Language Query, In Proc. ACMSIGIR Conf., pp.135-150.

Balabanovic, M. and Shoham, Y.. 1997. Fab: ContentBased, Collaborative Recommendation, Communications of the ACM, 40(3), pp.66-72.

Claypool, M., Gokhale, A., Miranda, T., Murnikov, P., Netes, D. and Sartin, M.. 1999. Combining contentbased and collaborative filters in an online newspaper, In Proc. ACM-SIGIR Workshop on Recommender Systems: Algorithms and Evaluation.

Delgado, J., Ishii, N. and Ura, T.. 1998. Content-based Collaborative Information Filtering: Actively Learning to Classify and Recommend Documents, In Proc. Second Int. Workshop, CIA'98, pp.206-215.

Douglas B. Terry. 1993. A tour through tapestry, In Proc. ACM Conf. on Organizational Computing Systems (COOCS). pp.21-30.

Gupta, D., Digiovanni, M., Narita, H. and Goldberg, K. 1999. Jester 2.0: A New Linear-Time Collaborative Filtering Algorithm Applied to Jokes, In Proc. ACMSIGIR Workshop on Recommender Systems: Algorithms and Evaluation.

Han, J., and Kamber, M.. 2000. Data mining: Concepts and Techniques. New York: Morgan-Kaufman.

Harman D.. 1994. Overview of TREC-3, In Proc.TREC3, pp.1-19.

Hauver, D. B.. 2001. Flycasting: Using Collaborative Filtering to Generate a Play list for Online Radio, In Int. Conf. on Web Delivery of Music.

Herlocker, J., Konstan, J., Borchers A., and Riedl, J.. 1999. An algorithmic framework for performing collaborative Filtering, In Proc. ACM-SIGIR Conf., 1999, pp. 230-237.

Kim, M. and Raghavan, V.V.. 2000. Adaptive conceptbased retrieval using a neural network, In Proc. Of
ACM-SIGIR Workshop on Mathematical/Formal Methods in IR.

McClave, J. T. and Dietrich, F. H.. 1998. Statistics. San Francisco: Ellen Publishing Company.

Lee, J.H., Kim, M.H. and Lee, Y.H.. 1993. Ranking documents in thesaurus-based Boolean retrieval systems, Information Processing and Management, 30(1), pp.79-91.

Oard, D.W. and Marchionini, G.. 1996. A conceptual framework for text filtering, Technical Report EETR-96-25, CAR-TR-830, CS-TR3643.

Ogawa, Y., Morita, T. and Kobayashi, K.. 1991. A fuzzy document retrieval system using the keyword connection matrix and a learning method, Fuzzy sets and Systems, 1991, pp.39, pp.163-179.

O'Conner, M. and Herlocker, J.. 1999. Clustering items for collaborative filtering, In Proc. ACM-SIGIR Workshop on Recommender Systems.

Resnick, P., Iacovou, N., Suchak, M., Bergstorm, P. and Riedl, J.. 1994. GroupLens: An open architecture for collaborative filtering of Netnews, In Proc. ACM Conf. on Computer-Supported Cooperative Work. pp.175-186.

Ricardo Baeza-Yates, Berthier Riberio-Neto. 1999. Modern Information Retrieval. New York:AddisonWesley Publishers.

Robertson S. E. and Sparck Jones K.. 1976. Relevance weighting of search terms, J. of the American Society for Information Science, 1976, pp.27, pp.129-146.

Salton, G. and Buckley, C.. 1988. Term-weight approaches in automatic retrieval, Information Processing and Management, 24(5), 1988, pp.513-523.

Sarwar, B. M., Karypis, G., Konstan, J. A. and Riedl, J.. 2001. Item-based Collaborative Filtering Recommendation Algorithms, In Proc. Tenth Int. WWW Conf. 2001, pp. 285-295.

Upendra, S. and Patti, M.. 1995. Social Information Filtering: Algorithms for Automating "Word of Mouth", In Proc. ACM CHI'95 Conf. on Human Factors in Computing Systems. pp.210—217.

Verhoeff, J., Goffman, W. and Belzer, J.. 1961. Inefficiency of the use of the boolean functions for information retrieval systems, Communications of the ACM, 4, pp.557--558, pp.594.

Wasfi, A. M. A.. 1999. Collecting User Access Patterns for Building user Profiles and Collaborative Filtering, In Int. Conf. on Intelligent User Interfaces. pp.57- 64 . 